\title{
Las Bioferias y su potencial para promover el consumo de productos agroecológicos en Quito
}

The Bioferias and its potential to promote the consumption of agroecological produce in Quito

\author{
Cristian Vasco ${ }^{1}$, Antonella Guevara ${ }^{2}$, Belen Jácome ${ }^{1}$, Diana Vasco Mora ${ }^{2}$ \\ ${ }^{1}$ Facultad de Ciencias Agricolas, Universidad Central del Ecuador, Quito, Ecuador, clvasco@uce.edu.ec \\ ${ }^{2}$ Universidad Técnica Estatal de Quevedo, Quevedo, Ecuador
}

Rec.: 15.01.2019. Acept.: 27.06.2019.

Publicado el 30 de junio de 2019

\section{Resumen}

$\mathrm{S}_{\mathrm{e}}^{\mathrm{e}}$ analizaron las motivaciones para consumir limentos agroecológicos en Quito, con datos de una encuesta realizada a los clientes de Bioferias entre abril y mayo de 2017. Los resultados revelan que los consumidores agroecológicos son sustancialmente diferentes del resto de la población en términos de educación e ingresos. La principal motivación para comprar en las Bioferias es que los productos agroecológicos se perciben como más saludables que los convencionales. Por otro lado, la preocupación por el medio ambiente es la motivación menos importante. Adicionalmente, los resultados de un modelo de regresión lineal muestran que los individuos educados, con mayores ingresos y que forman parte de una organización social/medioambiental son los que más gastan en productos agroecológicos.

Palabras clave: productos agroecológicos, Bioferias, motivaciones de consumo, gasto, Quito.

\begin{abstract}
$\mathrm{W}$ ith data from a survey conducted in April-May 2017 among the costumers of farmers' markets, we analyzed the motivations for the consumption of agroecological foodstuffs in Quito, Ecuador. The results reveal that agroecological consumers substantially differ from the rest of the population in terms of education and income. The principal motivations to buy in agroecological farmers' markets is that agroecological produce is perceived as healthier than conventionally produced food. On the other hand, environmental concern is the least important motivation to consume agroecological food. Additionally, the results of a linear regression model show that educated individuals with higher incomes that belong to a social/environmental are those who spend the most on agroecological products.
\end{abstract}

Keywords: agroecological products, farmers'Bioferias, motivations of consumption, expenditure, Quito. 


\section{Introducción}

E varias partes de Latinoamérica, movimientos sociales y organizaciones de agricultores han establecido ferias agroecológicas de agricultores como un espacio donde los productores pueden exhibir $\mathrm{y}$ vender sus productos y los consumidores pueden tener acceso a alimentos sanos a precios razonables (McKay y Nehring, 2014). Estas iniciativas han sido apoyados por organizaciones gubernamentales y no gubernamentales, como una estrategia para alcanzar equidad social, soberanía alimentaria y generar conciencia ambiental entre los productores (McKay y Nehring, 2014; Gomes et al, 2015;. Heifer International, 2015). Sin embargo, las ferias agroecológicas también surgien como una crítica a la agricultura orgánica, la que de acuerdo con algunos (Nelson et al., 2010; Buck et al., 1997; Guthman, 2002) ha hecho poco para limitar la participación de grandes compañías en el mercado de productos orgánicos, proteger a los pequeños productores de países en desarrollo, salvaguardar los derechos de los trabajadores agrícolas y restringir el monocultivo. Esto ha dado lugar a un "convencionalización del sector orgánico" (Nelson et al., 2010), la "elitización de productos sanos", y la distinción entre "mercados para ricos y mercados para pobres" (Intriago et al., 2017), con costos de la certificación orgánica prohibitivos para los pequeños productores y precios de productos certificados inasequibles para la mayoría de los consumidores en el mundo en desarrollo.

Dado que el futuro de la producción ecológica depende, en gran medida, de su capacidad para satisfacer las preferencias, necesidades y deseos de los consumidores (Lockie et al., 2002), un importante número estudios ha analizado las motivaciones para consumir alimentos orgánicos. En este sentido, varios estudios se han realizado en países desarrollados (Tanner y Wolfing Kast, 2003; Kvakkestad et al., 2018; O'Donovan y McCarthy, 2002; Lee y Yun, 2015; Lockie et al., 2004). Sin embargo, no se conoce de estudios empíricos que hayan analizado los determinantes del consumo agroecológico en los países menos desarrollados.

La literatura clasifica las motivaciones para consumir productos orgánicos en motivaciones del "bien público" o "altruistas" y motivaciones de "bien personal o "egoístas" (Bravo et al., 2013; OCDE, 2014). El primer término, bien personal, se refiere a los individuos que consumen alimentos orgánicos motivados principalmente por los beneficios personales asociados este. Estos incluyen, por ejemplo, las percepciones generalizadas de que los productos orgánicos son más saludables y tienen mejor sabor que los convencionales. En el caso de las motivaciones altruistas o de bien público, las personas compran alimentos orgánicos porque su consumo se percibe como amigable con el medio ambiente, respetuoso del bienestar animal, y, en menor medida, socialmente justo (Kvakkestad et al., 2018). En general, la percepción de que los productos orgánicos son más saludables son la principal motivación de consumo.

Varios estudios han analizado el efecto de las características individuales y de hogar en el gasto en alimentos orgánicos. Por ejemplo, estudios previos reportan efectos mixtos de la riqueza en el gasto en alimentos orgánicos. Algunos estudios reportan un efecto positivo (Bellows et al., 2008; Allender y Richards, 2010), mientras que otros (Zepeda y Li, 2007) concluyen que no existe ningún efecto. La educación, en tanto, tiene un efecto positivo sobre los gastos en alimentos orgánicos (Zepeda y Li, 2007; Bellows et al., 2008). Algunos estudios (Loureiro et al., 2001; Thompson y Kidwell, 1998) indican que los hogares con niños son más propensos a comprar alimentos orgánicos. Sin embargo, ¿aplican estos hallazgos para los productos agroecológicos y el concepto de Bioferias en los países en desarrollo? Además, ¿tienen los mercados de agricultores el potencial para cumplir el objetivo de hacer que la alimentación saludable esté al alcance de la generalidad de la población?

Con datos de una encuesta realizada entre los consumidores de las Bioferias en Quito, este trabajo examina las motivaciones de consumo de productos agroecológicos y los determinantes del gasto en alimentos agroecológicos.

\section{La agroecología y mercados agroecológicos en Ecuador}

Al igual que en la mayoría de los países de América Latina, la agroecología en Ecuador surgió en la década de 1980 como una respuesta a la degradación ambiental resultante de la revolución verde. En este contexto, varias ONGs y asociaciones de agricultores emergieron para rescatar a los conocimientos ancestrales de los agricultores locales, centrándose no sólo en los aspectos técnicos y económicos, sino también la incorporación de las dimensiones sociales, culturales y ambientales de la producción agrícola (Macas y Echarry, 2009; Heifer International, 2015). Si bien la definición de la agroecología puede variar de una organización a otra, la mayoría de ellas se centran en la mitigación de los problemas ambientales resultantes del uso de pesticidas y fertilizantes químicos en la agricultura convencional; y la integración de las dimensiones sociales, económicas y culturales de la agricultura;

Dado que la mayoría de los productores 
agroecológicos tienen poca tierra y no tienen acceso a supermercados, la comercialización de productos agroecológicos se produce normalmente en el marco de esquemas de comercialización alternativos que incluyen Bioferias, canastas de alimentos, mercados de trueque y tiendas agroecológicas, siendo las Bioferias la forma de comercialización que más importante (Intriago et al., 2017; Heifer International, 2015). El contacto directo entre el cliente y el productor mantiene a los intermediarios fuera de la cadena de comercialización y por tanto, permite a los agricultores acceder a un mayor beneficio. Otra ventaja de las Bioferias es que las mismas están organizadas por asociaciones de agricultores, y por tanto, todos los miembros pueden participar sin importar las cantidades que son capaces de suministrar.

En 2002, el Municipio de Quito inició el proyecto AGRUPAR (Agricultura Urbana Participativa). Esta iniciativa se centra en la reducción de la pobreza y en mejorar la seguridad alimentaria entre los residentes de áreas urbanas, periurbanas y rurales de la ciudad de Quito, dando prioridad a los grupos tradicionalmente marginados, como mujeres, madres solteras, ancianos, los inmigrantes recién llegados, poblaciones indígenas, desempleados, entre otros (Rodríguez y Proaño, 2016; Anguelovski, 2009). De hecho, la mayoría de los miembros son mujeres $(84 \%)$ con fincas de menos de $600 \mathrm{~m}^{2}$ (Rodríguez y Proaño, 2016). Como parte del proyecto, AGRUPAR entrenó a los agricultores en la producción agroecológica, el emprendimiento y gestión y comercialización. Aunque en un principio el proyecto iba dirigido a mejorar la seguridad alimentaria, promover la conciencia ambiental y la producción y el consumo de alimentos sanos entre los residentes pobres de Quito, los excedentes son comercializados en 14 Bioferias que se organizan semanalmente por AGRUPAR (Quinga, 2016; Mena Pérez, 2012). La transparencia y la calidad de los productos comercializados en las Bioferias están garantizadas por un Sistema de Garantía Participativa (SPG), la cual se basa en relaciones de confianza entre productores y consumidores (Rodríguez y Proaño Rivera, 2016).

Los ingresos de los excedentes comercializados en Bioferias ayudan a equilibrar los ingresos familiares de los productores agroecológicos, con un ingreso promedio mensual de ventas de US $\$ 130$, una cantidad que representa el 36\% del salario mínimo de Ecuador (Oviatt, 2016). Otro objetivo del proyecto AGRUPAR es promover el consumo de alimentos agroecológicos a precios asequibles entre la población urbana, principalmente entre aquellos que, de lo contrario, no serían capaces acceder a alimentos saludables (Rodríguez y Proaño, 2016; Mena, 2012).

\section{Métodos}

\section{La encuesta}

Los datos proceden de una encuesta realizada entre los compradores de las Bioferias organizadas por AGRUPAR en el Distrito Metropolitano de Quito. El cuestionario incluyó información sobre las características demográficas de los compradores (edad, sexo, escolaridad de nivel, los ingresos personales, tipo de ocupación), características del hogar (composición de los hogares, el ingreso total del hogar, propiedad de la vivienda, zona de residencia), los hábitos personales (actividad física y la pertenencia a una organización). Otra sección inquirió a los encuestados acerca de la razón principal para consumir productos agroecológicos, con las opciones de: salud, características del producto (aspecto y sabor), conciencia ambiental y el apoyo a los productores como opciones de respuesta.

Es preciso señalar que en este estudio no fue posible estimar una muestra probabilística, dado que no se disponía del universo de compradores de las ferias agroecológicas. En ausencia de esta información, se recogió una muestra lo suficientemente grande y diversa para reflejar las características más importantes de la población de los compradores en Bioferias. Para lograr este propósito, los entrevistados fueron seleccionados utilizando un método de muestreo de dos etapas. En primer lugar, con el fin de garantizar una amplia diversidad de clientes, nueve de las 14 Bioferias fueron incluidos en la muestra. La encuesta fue realizada entre abril y mayo de 2017 por estudiantes de pregrado. Se obtuvo una muestra de 254 individuos.

Dado que los mercados están dispersos por toda la ciudad, incluyendo los barrios ricos y pobres, en el centro y las afueras de la ciudad, se aseguró la variabilidad de los datos en términos de ingresos y origen étnico de los clientes. A continuación, la encuesta se administró al azar a los clientes dentro de cada mercado seleccionado, es decir, todos los clientes adultos tenían la misma probabilidad de ser parte de la muestra, lo que aseguró variabilidad en cuanto a edad, educación y género.

\section{Análisis estadísticos}

Para establecer los determinantes del gasto en productos agroecológicos, se utilizó el método de regresión lineal por mínimos cuadrados ordinarios (MCO), en la que la cantidad mensual gastada en frutas y verduras fue una función de las características individuales y del hogar del encuestado. En definitiva, se utilizó un modelo de la siguiente forma:

$$
Y_{i}=\beta \mathrm{X}_{\mathrm{i}}+\mu_{\mathrm{i}}
$$

donde $Y$ es el logaritmo natural del gasto mensual en productos agroecológicos del individuo i, $\mathrm{X}$ es un vector 
de características individuales y de hogar, las cuales se describen más adelante, $\beta$ es un vector de coeficientes cuya dirección y magnitud son de interés en este estudio y $\mu$ es el error.

Las variables explicativas incluyeron grupos de características individuales y del hogar. En términos de variables individuales se incluyeron la edad, el género, los años de educación formal y el estado civil del comprador. El logaritmo natural del ingreso del individuo se utilizó como un indicador de la riqueza del individuo. Adicionalmente, tres variables dicotómicas que tomaron el valor de 1 si el entrevistado declara: hacer ejercicio con regularidad, ser miembro de una organización y ser vegetariano, respectivamente, controlaron los efectos de estilo de vida del comprador en el gasto en productos agroecológicos. A nivel del hogar, se incluyeron el tamaño del hogar y el número de niños (personas menores de 15 años).

Finalmente, tres variables dicotómicas que toman el valor de 1 si el individuo compró alimentos agroecológicos motivada principalmente por las características del producto (aspecto y sabor), intención de apoyar a los productores locales o conciencia ambiental, respectivamente, se incluyeron en el modelo. El grupo de personas que compran productos agroecológicos debido a que los asocian alimentos saludables -el más numeroso en la muestrase dejó como el grupo de referencia (comparación).

\section{Resultados}

Características de los consumidores agroecológicos

En la Cuadro 1 se comparan varias características demográficas y socioeconómicas de los consumidores de productos agroecológicos con los de la población de Quito en general. Para este efecto, se utilizaron datos del Censo Nacional-2010 (INEC, 2010), la Encuesta de Empleo, Subempleo y Desempleo 2016 (INEC, 2016), Encuesta de Uso del Tiempo-2012 (INEC, 2012) y la Encuesta de Condiciones de Vida 2013-2014 (INEC, 2014).

En promedio, los compradores de las Bioferias exhiben mayores niveles de educación formal que el resto de la población de Quito. Casi la mitad de los consumidores agroecológicos $(48.9 \%)$ poseen un título universitario, mientras que solo el $32 \%$ de la población general de Quito ostenta un título universitario. Del mismo modo, la fracción de los consumidores que poseen título de postgrado (13.3\%) es cuatro veces más alta que la de la media de estimada para Quito. El hogar promedio de un comprador agroecológico es ligeramente menor que el promedio de los hogares en Quito. Sin embargo, los hogares que consumen productos agroecológicos tienen el doble de niños (personas menores de 15 años) que el hogar típico en Quito. El ingreso mensual promedio de los consumidores agroecológicos (US \$ $1,081)$ es tres veces más alto que el de un ciudadano promedio de Quito en edad de trabajar (US \$ 381). La

Cuadro 1. Comparación entre los consumidores agroecológicos y la población de Quito en general.

\begin{tabular}{lll}
\hline \multicolumn{1}{c}{ Variable } & \multicolumn{1}{c}{ Individuos en la muestra } & Población de Quito en general \\
\hline Nivel educativo (\%) & & \\
Iletrado & 0.0 & $2.5^{a}$ \\
Educación de primaria & 5.0 & $22.9^{a}$ \\
Educación secuandaria & 32.8 & $23.7^{a}$ \\
Educación superior & 48.9 & $32.1^{a}$ \\
Posgrado & 13.3 & $3.4^{a}$ \\
Tamaño del hogar & 3.2 & $3.5^{a}$ \\
Número de niños & 0.6 & $0.3^{a}$ \\
Ingreso promedio & 1081 & $381^{b}$ \\
Pertenece a organización (\%) & 19 & $1.1^{c}$ \\
Hace ejercicio (\%) & 29 & $16.5^{d}$ \\
\hline
\end{tabular}

a Calculado con los datos del Censo Nacional 2011

b Calculado con los datos del empleo y Encuesta sobre el desempleo-2016

c Calculado con datos de la Encuesta de Niveles de Vida de 2013

d Calculado con datos de la Encuesta de Empleo del Tiempo-2011 
fracción de compradores agroecológicos que son parte de una organización social (19\%) es considerablemente más alto que la de la población general $(1 \%)$. Por último, la fracción de los clientes de los mercados de los agricultores que hacen ejercicio regularmente es dos veces más alta que la del ciudadano medio de Quito.

\section{Motivaciones para consumir productos agroecológicos}

La Figura 1 muestra las motivaciones para comprar productos agroecológicos por categoría de ingresos para una muestra de 254 compradores. Los resultados muestran que las motivaciones personales son más importantes que las motivaciones altruistas en las decisiones de consumo. La salud es la principal motivación para comprar en Bioferias, con la mayoría de los encuestados (90\%) comprando productos agroecológicos debido a que se perciben como más saludables que los producidos convencionalmente. Una pequeña parte de los encuestados (5.6\%) compran productos agroecológicos debido a sus características (sabor y apariencia). En tanto, una fracción pequeña (4.6\%) de los entrevistados consumen alimentos agroecológicos debido a motivaciones altruistas (apoyo a los productores y conciencia ambiental).

Aunque la salud sigue siendo la principal motivación para consumir alimentos agroecológicos, al analizar las motivaciones por categoría de ingresos, hay algunos cambios que merecen ser mencionados. Las motivaciones altruistas parecen ser más importantes para el grupo de compradores con ingresos altos (más de US $\$ 2,000$ por mes), con el $10.7 \%$ de los encuestados argumentando que "el apoyo a los productores" es su principal motivación para comprar las Bioferias. Por el contrario, casi todos los encuestados con ingresos medianos (US \$ 1,000- 2,000 al mes) compran alimentos agroecológicos debido a que se perciben

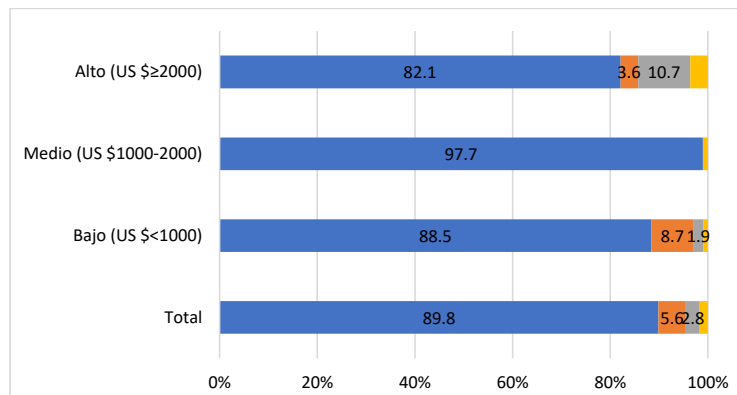

$\square$ Salud $\square$ Características del producto $\square$ Apoyo a productores $\llbracket$ Conciencia ambiental

Figura 1. Motivaciones para consumir productos agroecológicos por categoría de ingresos de los compradores de productos agroecológicos en Quito (2017). como saludables. En el caso de los compradores con ingresos bajos ( $<$ US $\$ 1,000$ por mes), estos están más preocupados de las características del producto que sus pares con medianos y altos ingresos.

La Figura 2 muestra las motivaciones para comprar en Bioferias por el gasto mensual en productos agroecológicos. Los encuestados con gastos bajos $(<\mathrm{US}$ $\$ 100$ al mes) parecen ser los más preocupados por la inocuidad de los alimentos que consumen, con un 94\% de los individuos en este grupo comprando productos agroecológicos debido a que se asocian con alimentos sanos. Por el contrario, el $8.3 \%$ de los individuos con gastos medios (US \$ 100-300 al mes) consumen productos agroecológicos debido a su apariencia $\mathrm{y}$ sabor.

\section{Determinantes del gasto en productos agroecológicos}

Las definiciones y estadísticas descriptivas utilizadas para el análisis se presentan en el Cuadro 2. Como mencionó anteriormente, se utilizó el logaritmo natural del gasto mensual en productos agroecológicos como la variable dependiente.

El Cuadro 3 presenta los resultados del modelo de regresión lineal. Los resultados muestran que, en promedio, las mujeres gastan un $27 \%$ menos en los productos agroecológicos que los hombres. La educación tiene un efecto positivo sobre el consumo de alimentos agroecológicos. Cada año de educación formal aumenta el gasto en productos agroecológico en un $4.9 \%$. Los individuos casados gastan un $43 \%$ menos que sus pares solteros. En promedio, los encuestados que forman parte de una organización social gastan un $37 \%$ más que los que no lo están.

Los individuos de hogares con más niños gastan más en alimentos agroecológicos. Cada niño en el hogar aumenta el gasto en alimentos agroecológicos en un $26 \%$. En cuanto a las motivaciones para comprar en

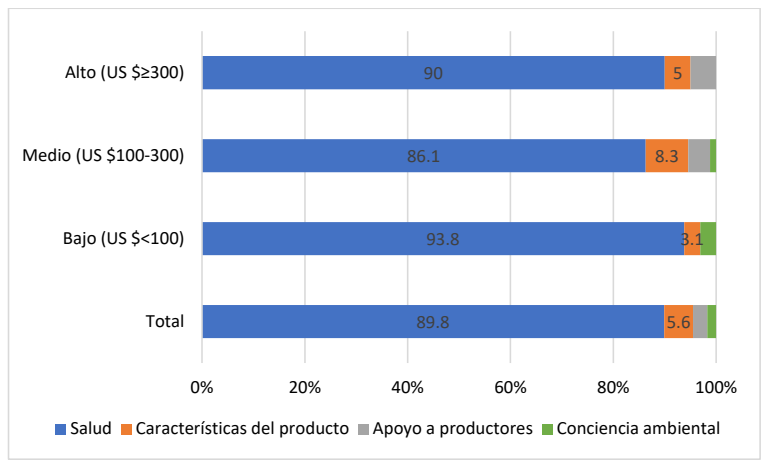

Figura 2. Motivaciones para comprar en Bioferias por el gasto mensual en productos agroecológicos de los compradores de productos agroecológicos en Quito (2017). 
Cuadro 2. Estadísticas descriptivas y definiciones de variables de los compradores de productos agroecológicos en Quito (2017).

\begin{tabular}{|c|c|c|c|}
\hline Variable & Descripción & Media & Desv. Stand. \\
\hline \multicolumn{4}{|l|}{ Variables dependientes } \\
\hline Gasto & Gasto mensual en productos agroecológicos (US \$) & 196.2 & 182.6 \\
\hline \multicolumn{4}{|c|}{ Variables independientes } \\
\hline Edad & Edad del individuo (años) & 45.3 & 14.8 \\
\hline Mujer $(0 / 1)$ & El individuo es mujer & 0.6 & - \\
\hline Educación & Años de educación formal & 16.5 & 3.9 \\
\hline Casado $(0 / 1)$ & El Individuo está Casado & 0.46 & - \\
\hline Ingresos & Ingresos mensuales (US \$) & 1081.8 & 1027.5 \\
\hline Ejercicio $(0 / 1)$ & El individuo hace ejercicio regularmente & 0.33 & - \\
\hline Vegetariano $(0 / 1)$ & El individuo es vegetariano & 0.11 & - \\
\hline Asociación $(0 / 1)$ & El individuo es miembro de una organización & 0.22 & - \\
\hline Tamaño del hogar & Número de miembros del hogar & 3.15 & 1.35 \\
\hline Niños & Número de niños en el hogar & 0.54 & 0.80 \\
\hline Salud $(0 / 1)$ & $\begin{array}{l}\text { La salud es la principal motivación para consumir } \\
\text { alimentos agroecológicos }\end{array}$ & 0.90 & - \\
\hline Características $(0 / 1)$ & $\begin{array}{l}\text { Las características del producto son la principal } \\
\text { motivación para consumir alimentos agroecológicos }\end{array}$ & 0.06 & - \\
\hline Apoyo $(0 / 1)$ & $\begin{array}{l}\text { Apoyo a los agricultores es la principal motivación para } \\
\text { consumir alimentos agroecológicos }\end{array}$ & 0.03 & - \\
\hline Medio ambiente $(0 / 1)$ & $\begin{array}{l}\text { La preocupación ambiental es la principal motivación } \\
\text { para consumir alimentos agroecológicos }\end{array}$ & 0.01 & - \\
\hline
\end{tabular}

Nota: $(0 / 1)$ identifica variables dicotómicas.

Cuadro 3: Determinantes del gasto en productos agroecológicos por el método de mínimos cuadrados ordinarios (MCO).

\begin{tabular}{ll}
\hline Variable & Coefficients \\
\hline Edad & 0.002 \\
Edad al cuadrado & 0.000 \\
Mujer $(0 / 1)$ & $-0.235^{* *}$ \\
Educación & $0.049 * * *$ \\
Casado $(0 / 1)$ & $-0.357 * * *$ \\
Ingresos & $0.068 * *$ \\
Ejercicio $(0 / 1)$ & -0.068 \\
Vegetariano $(0 / 1)$ & 0.324 \\
Asociación $(0 / 1)$ & $0.316 * *$ \\
Tamaño del hogar & -0.001 \\
Niños & $0.262 * * *$ \\
Características $(0 / 1)$ & 0.060 \\
Apoyo $(0 / 1)$ & $0.468 * *$ \\
Medio ambiente $(0 / 1)$ & 0.081 \\
\hline Prob $>\mathrm{F}$ & $\mathbf{6 9}$ \\
Número de observaciones & $\mathbf{2 5 4}$ \\
$\mathrm{R}^{2}$ & $\mathbf{0 . 3 8}$ \\
Nota: *** y $* * *$ & denotan \\
significación estadística al 10,5 & \\
y 1\%, respectivamente. & \\
\hline
\end{tabular}

Bioferias, solo la variable dicotómica que indica que un individuo consume productos agroecológicos debido a que de esta manera apoya a los productores urbanos, tiene un efecto significativo.

\section{Discusión}

A unque las Bioferias han emergido como una - Aalternativa para el consumo de ecológico entre la población local, es decir, entre los que de otra manera no serían capaces de costear alimentos saludables (Intriago et al., 2017; Heifer Internacional 2015), los resultados de este estudio indican que los consumidores agroecológicos pertenecen a un grupo de individuos que difieren sustancialmente de la población promedio en términos de sus características socioeconómicas. Son más ricos, mejor educados, y provienen de hogares más pequeños. Además, a diferencia de la residente promedio de Quito, muchos individuos de este grupo pertenecen a una organización social.

El que los consumidores agroecológicos ostenten mejor educación que la población media de Quito podría reflejar que están más conscientes de los peligros del consumo los productos convencionales y los beneficios 
asociados con el consumo de alimentos agroecológicos (Zepeda y Li, 2007). Del mismo modo, el que los consumidores agroecológicos tengan mayores ingresos que el habitante promedio de Quito podría indicar que estos son capaces de costear el precio más alto de los productos agroecológicos (Bellows et al., 2008; Allender y Richards 2010). Si bien estos resultados son consistentes con los reportados para el consumo de productos orgánicos (OCDE, 2014), los mismos reflejan que, lejos de ser disponible para el común de la población, la producción agroecológica es consumida por un segmento de la población con características especiales en cuanto a ingresos, educación y estilo de vida.

Los resultados indican que la principal motivación para comprar productos agroecológicos es que estos se asocian con alimentación saludable. Este hallazgo era predecible, ya que la salud es la principal motivación para consumir alimentos orgánicos en los países desarrollados (Lockie et al., 2002, Schifferstein y Ophuis, 1998; Gracia y De Magistris, 2008; O’Donovan y McCarthy, 2002; Tregear et al., 1994; Kvakkestad et al., 2018; Squires et al., 2001; Durham y Andrade, 2005). Sin embargo, es preciso señalar que la proporción de encuestados que compra productos agroecológicos debido a que los considera más saludables que los convencionales $(90 \%$ de la muestra) es sustancialmente más alta que la reportada para los consumidores orgánicos en países desarrollados (50\%) (OCDE, 2014; Boccaletti, 2009).

En relación a la pregunta de ¿quién gasta más en las Bioferias? Los resultados muestran que este es un hombre bien educado y con altos ingresos. Este individuo es más propenso a ser parte de una organización social o ambiental y a comprar productos agroecológicos motivado por el deseo de apoyar a los pequeños productores. El hecho de que los individuos más ricos y educados gasten más en productos agroecológicos puede reflejar que estas personas conocen más acerca de los riesgos del uso de plaguicidas en la producción de alimentos convencionales y tienen los recursos para costear una alimentación agroecológica en comparación con las personas más pobres (Andrade y Flores, 2008). El efecto positivo y significativo de la pertenencia a una organización social / ambiental puede estar asociada con una mayor preocupación por los problemas sociales y ambientales y, por tanto, con una mayor predisposición a pagar un precio más alto por lo que se considera amigable con el medio ambiente y socialmente justo (OCDE, 2014). Los resultados también muestran que los individuos de hogares con más niños gastan más en productos agroecológicos. Esto es consistente investigaciones previas sobre los determinantes del consumo de alimentos orgánicos (Loureiro et al.,
2001; Kiesel y Villas-Boas, 2007) y puede reflejar que los compradores con niños prestan más atención a la inocuidad de los productos con que alimentan a sus hijos. Otro hallazgo interesante es que los individuos cuya principal motivación para consumir productos agroecológicos es el apoyo a los pequeños agricultores gastan más que aquellos cuya principal motivación de compra es la salud.

¿Estos resultados apoyan la idea de que la promoción y diseminación de las Bioferias puede ser una estrategia eficaz para masificar el consumo saludable la población local que no puede pagar productos orgánicos certificados? Al parecer aún no. Con solo un selecto grupo de individuos educados y con buenos ingresos que compran productos agroecológicos, las Bioferias todavía no han cumplido con su objetivo de masificar el consumo de alimentos saludables.

En este sentido, las políticas deben estar orientadas a la publicitar más los beneficios del consumo de alimentos agroecológicos entre la población, principalmente entre aquellos con bajos niveles de educación e ingresos. Los resultados presentados en este estudio deberían ser una llamada de atención para los responsables de las políticas encaminadas a incrementar la conciencia ambiental entre los ciudadanos, ya que la preocupación por el medio ambiente se ubica como la motivación menos importante para comprar alimentos agroecológicos. En este sentido, las campañas de promoción del consumo agroecológico deben ir más allá del atributo "salud" de los productos agroecológicos. En cambio, los consumidores potenciales también deben conocer que, mediante el consumo de productos agroecológicos (en las Bioferias), también estarían contribuyendo al uso sostenible de los recursos naturales y a mejorar las condiciones de vida de los pequeños productores.

\section{Conclusiones}

$\mathrm{E}$ este trabajo se analizaron las motivaciones para consumir productos agroecológicos en Quito. Los consumidores de productos agroecológicos difieren sustancialmente del resto de la población en términos de su educación, ingresos y estilo de vida. Para los consumidores de productos agroecológicos, la alimentación saludable es, por mucho, la principal motivación para comprar en Bioferias. Por el contrario, la conciencia ambiental parece no tener influencia en las decisiones de consumo agroecológico.

Desde varios sectores se argumenta que las Bioferias tienen el potencial de promover el consumo de alimentos saludables al mismo tiempo que mejoran las condiciones de vida de los pequeños productores con y promueven una agricultura sostenible. Los hallazgos 
aquí presentados indican, sin embargo, que lejos de ser accesible para todos, los productos agroecológicos son consumidos principalmente por un grupo de individuos educados y con buenos ingresos que no están realmente preocupados por los potenciales beneficios para el medio ambiente que conlleva la producción agroecológica. Por lo tanto, se requieren esfuerzos adicionales para que la producción agroecológica sea accesible para todos.

\section{Bibliografía}

Allender, W. J., and T. J. Richards. 2010. Consumer Impact of Animal Welfare Regulation in the California Poultry Industry. Journal of Agricultural and Resource Economics: 424-442.

Andrade, D., and M. Flores. 2008. Consumo de productos orgánicos/agroecológicos en los hogares ecuatorianos. Quito, Ecuador: VECO.

Anguelovski, I. 2009. Building the Resilience ofVulnerable Communities inQuito: Adapting local foodsystems to climate change. Urban Agriculture 22:25-26.

Bellows, A. C., B. Onyango, A. Diamond, and W. K. Hallman. 2008. Understanding consumer interest in organics: production values vs. purchasing behavior. Journal of Agricultural \& Food Industrial Organization 6(1):1-31.

Boccaletti, S. 2009. Organic Food Consumption: Results and Policy Implications. In Household Behaviour and Environmental Policy. Paris.

Bravo, C. P., A. Cordts, B. Schulze, and A. Spiller. 2013. Assessing determinants of organic food consumption using data from the German National Nutrition Survey II. Food Quality and Preference 28(1):60-70.

Buck, D., C. Getz, and J. Guthman. 1997. From farm to table: The organic vegetable commodity chain of Northern California. Sociologia ruralis 37(1):3-20.

Durham, C.A., and D. Andrade. 2005. Health vs. environmental motivation in organic preferences and purchases. Paper read at American Economics Association Annual Meeting.

Gomes, R. A., T. L. Matias, and J. S. Paulino. 2015. Articulações interinstitucionais na realização de feiras agroecológicas na Microrregião de Campina GrandePB. Revista Brasileira de Desenvolvimento Regional 3(1):103-126.

Gracia, A., and T. De Magistris. 2008. The demand for organic foods in the South of Italy: A discrete choice model. Food Policy 33(5):386-396.

Guthman, J. 2002. Commodified meanings, meaningful commodities: Re-thinking production-consumption links through the organic system of provision. Sociologia ruralis 42(4):295-311.

Heifer International. 2015. La agroecología está presente: Mapeo de productores agroecológicos y del estado de la agroecología en la sierra y costabecuatoriana. Quito,
Ecuador: Heifer-Ecuador.

INEC. 2010. Censo de Población y Vivienda-2010.

2012. Encuesta de Uso del Tiempo-2012.

2014. Encuesta de Condiciones de Vida 2014-2015, edited by I. N. d. E. y. Censos. Quito, Ecuador.

Encuesta de Empleo, Subempleo y Desempleo 2016 [cited. Available from http://www.ecuadorencifras.gob.ec/ enemdu-2016/.

Intriago, R., R. Gortaire Amézcua, E. Bravo, and C. O'Connell. 2017. Agroecology in Ecuador: historical processes, achievements, and challenges. Agroecology and Sustainable Food Systems 41(3-4):311-328.

Kiesel, K., and S. B. Villas-Boas. 2007. Got organic milk? Consumer valuations of milk labels after the implementation of the USDA organic seal. Journal of agricultural \& food industrial organization 5(1).

Kvakkestad, V., H. Berglann, K. Refsgaard, and O. Flaten. 2018. Citizen and consumer evaluation of organic food and farming in Norway. Organic Agriculture:1-17.

Lee, H.-J., and Z.-S. Yun. 2015. Consumers' perceptions of organic food attributes and cognitive and affective attitudes as determinants of their purchase intentions toward organic food. Food quality and preference 39:259-267.

Lockie, S., K. Lyons, G. Lawrence, and J. Grice. 2004. Choosing organics: a path analysis of factors underlying the selection of organic food among Australian consumers. Appetite 43(2):135-146.

Lockie, S., K. Lyons, G. Lawrence, and K. Mummery. 2002. Eating 'green': motivations behind organic food consumption in Australia. Sociologia ruralis 42(1):2340.

Loureiro, M. L., J. J. McCluskey, and R. C. Mittelhammer. 2001. Assessing consumer preferences for organic, ecolabeled, and regular apples. Journal of agricultural and resource economics:404-416.

Macas, B., and K. Echarry. 2009. Caracterización de mercados locales agroecológicos y sistemas participativos de garantía que se construyen en el Ecuador. In Quito: Coordinadora Ecuatoriana de Agroecología.

McKay, B., and R. Nehring. 2014. Sustainable agriculture: An assessment of Brazil's family farm programmes in scaling up agroecological food production: Working Paper, International Policy Centre for Inclusive Growth.

Mena Pérez, V. E. 2012. Evaluación integral del programa AGRUPAR de Conquito correspondiente al Distrito Metropolitano de Quito, Universidad Central del Ecuador.

Nelson, E., L. G. Tovar, R. S. Rindermann, and M. Á. G. Cruz. 2010. Participatory organic certification in Mexico: an alternative approach to maintaining the integrity of the organic label. Agriculture and Human Values 27(2):227237.

O'Donovan, P., and M. McCarthy. 2002. Irish consumer 
preference for organic meat. British Food Journal 104(3/4/5):353-370.

OECD. 2014. Greening Household Behaviour: OECD.

Oviatt, K. 2016. "El impacto de la Agricultura Urbana como método de empoderamiento de las poblaciones pobres: University of Denver.

Quinga Guallichico, T. M. 2016. Evaluación y determinación del estado del proyecto de agricultura urbana participativa Agrupar de Conquito en el distrito metropolitano de Quito. Tesis para optar al título de Ingeniero Agrónomo, Universidad Central del Ecuador.

Rodríguez Dueñas, A., and I. Proaño Rivera. 2016. Quito siembra agricultura urbana. Quito, Ecuador: CONQUITO-AGRUPAR.

Squires, L., B. Juric, and T. Bettina Cornwell. 2001. Level of market development and intensity of organic food consumption: cross-cultural study of Danish and New Zealand consumers. Journal of Consumer Marketing 18(5):392-409.

Tanner, C., and S. Wölfing Kast. 2003. Promoting sustainable consumption: Determinants of green purchases by Swiss consumers. Psychology \& Marketing 20(10):883-902.

Thompson, G. D., and J. Kidwell. 1998. Explaining the choice of organic produce: cosmetic defects, prices, and consumer preferences. American journal of agricultural economics 80(2):277-287.

Tregear, A., J. Dent, and M. McGregor. 1994. The demand for organically grown produce. British Food Journal 96(4):21-25.

Zepeda, L., and J. Li. 2007. Characteristics of organic food shoppers. Journal of Agricultural and Applied Economics 39(1):17-28. 